\title{
Improved High Performance Recycling of Polymers by Means of Bi-Exponential Analysis of Their Fluorescence Lifetimes
}

\author{
Heinz Langhals*, Dominik Zgela, Thorben Schlücker \\ Department of Chemistry, LMU University of Munich, Munich, Germany \\ Email: ${ }^{*}$ Langhals@Irz.uni-muenchen.de
}

Received 2 April 2015; accepted 9 May 2015; published 14 May 2015

Copyright (C) 2015 by authors and Scientific Research Publishing Inc.

This work is licensed under the Creative Commons Attribution International License (CC BY). http://creativecommons.org/licenses/by/4.0/

cc) (i) Open Access

\begin{abstract}
Technical polymers could be identified by means of their remarkably strong auto fluorescence. The mono-exponentially obtained time constants of fluorescence decay were applied for a rough assignment of the polymeric materials whereas bi-exponential analysis allowed a fine classification such as for special batches and for preceding contaminations. Chemically similar materials such as LDPE (low-density polyethylene), HDPE (high-density polyethylene) and UHDPE (ultrahigh-density polyethylene) could be as well identified as contaminations of mineral oil in PET (polyethylene terephthalate). Furthermore, the fluorescence spectra could be characterized by means of five Gaussian functions in the visible allowing a redundant assignment to the fluorescence lifetimes. Thus, efficient sorting of polymers was possible for high performance recycling.
\end{abstract}

\section{Keywords}

Recycling, Polymers, Fluorescence Decay, Fluorescence Spectroscopy, Gaussian

\section{Introduction}

There is an increasing need for the recycling of organic polymers (plastics) both for economy and avoiding environmental pollution. The pacific trash vortex is one of the most prominent and impressive examples for the latter and even gets the name Great Pacific Garbage Patch. The majority of technical polymers are thermoplasts and may be re-used by melt and moulding again. However, the immiscibility and the incompatibility of different organic polymers are therefore the main obstacle, because high performance materials require uniformity where

"Corresponding author.

How to cite this paper: Langhals, H., Zgela, D. and Schlücker, T. (2015) Improved High Performance Recycling of Polymers by Means of Bi-Exponential Analysis of Their Fluorescence Lifetimes. Green and Sustainable Chemistry, 5, 92-100. 
a content of external material as low as $5 \%$ downgrades the value of polymers appreciably. As a consequence, pure polymers are required for an efficient recycling where a sorting becomes necessary for mixed collected materials. Sorting is also of interest for chemical processing of polymers because such processes operate most stable with uniform starting materials. The machine-based recognition of polymers is a prerequisite for such processes where methods using the density or electrostatic properties are described [1]-[3]. Optical methods [4] [5] are more attractive because of simple, stable and efficient technology where fluorescence is advantageous [6]-[10] because of unproblematic light path and detection. The doping of polymers with fluorescent markers [10] and their re-identification by the spectral resolution of their fluorescence in combination with a binary coding is described in preceding papers [11] [12], however, a secure pre-treatment of the polymers is a prerequisite for such methods. The use of the remarkably strong autofluorescence of polymers [13] is an attractive alternative because no pre-treatment is necessary and even the workup of deposited material will become possible.

\section{Materials and Methods}

Spectroscopy: Fluorescence spectra: Varian Cary Eclipse; fluorescence lifetimes: PicoQuant FluoTime 300; Pico Quant PicoHarp 300 (PC-405 laser; 403 nm).

The preferred wavelengths for the detection of the fluorescence decays were located by means of lifetime dependant fluorescence spectra. Starting at the maximum of the fluorescence intensity $I_{(t)}$ with $t=0$ and a delay of 3 ns the fluorescence spectrum was recorded with sampling until $100 \mathrm{~ns}$. The maximum $\lambda_{\max }$ of this fluorescence spectrum was used for the determination of the fluorescence lifetimes $\tau$ because of an amplification of slower component of decay (the spectrum of the shorter period between 0 and 3 ns was recorded for comparison). The fluorescence intensities were accumulated with a repetition rate of $20 \mathrm{MHz}$ during $10 \mathrm{~s}$; slightly increased noise is obtained for a shortened accumulation time of $1.0 \mathrm{~ms}$. The fluorescence lifetimes were obtained by exponential fitting (exponential tail fit) of the respective sections according to the equation $I_{(t)}=\mathrm{A} \cdot \mathrm{e}^{-t / \tau}$ by means of the software FluoFit from PicoQuant. The shape of the laser pulse was neglected due to its small FWHM. The decay time $\tau_{1}$ was calculated from the period between 0 and 3 ns and the decay time $\tau_{2}$ between 3 and 43 ns. Thus, all values are relative and are not independent on the used analytical equipment.

Materials: The technical polymers Luran ${ }^{\circledR}$ (styrene, polyacrylonitile copolymer from BASF), Delrin ${ }^{\circledR}$ (polyoxymethylene from DuPont) and Ultramid ${ }^{\circledR}$ (polyamide with glass fibre from BASF) were applied without further treatment. Spectroscopic grade solvents were applied. Tectosil ${ }^{\circledR}$ from Wacker Chemie AG; PET (polyethylene terephthalate) flakes from Inter Recycling $\mathrm{GmbH}$, applied without further purification.

Purification of PET: PET-Flakes were treated with stirring with a mixture of $3 \%$ aqueous $\mathrm{NaOH}(100 \mathrm{~mL})$ and $15 \%$ aqueous sodiumdodecylsulphate solution (SDS, $50 \mathrm{~mL}$ ) at $85^{\circ} \mathrm{C}$ for $2 \mathrm{~h}$, washed with distilled water, dried in air at room temperature and then at $60^{\circ} \mathrm{C}$ for $16 \mathrm{~h}$.

\section{Results and Discussion}

\subsection{First Order Decay of Autofluorescence}

The spectra of the autofluorescence of polymers are obtained with optical excitation in the UV, such as with common light sources at $365 \mathrm{~nm}$ or at short wavelengths in the visible region such as with well-available lasers at $403 \mathrm{~nm}$. The majority of the fluorescence emission extends in the visible at short wavelengths. The fluorescence decay can be mainly described with a first-order exponential function and the lifetime $\tau$ can be precisely determined and is characteristic for the polymeric material [13]. The special advantage of first order is the independence of the time constant as well from the initial intensity as the starting time of the measurement. The thus obtained time constants are reported for typical technical polymers in Figure 1.

Here we see that there is a broad range of $\tau$ between 0.2 until 5 ns where the individual time constants are as characteristic as fingerprints. Remarkably, the chemically very similar and otherwise difficult to discriminate types of polyethylene, LDPE (low-density polyethylene), HDPE (high-density polyethylene) and UHDPE (ultrahigh-density polyethylene) can be unambiguously distinguished; this may be a consequence of different microscopic rigidities of these materials. Moreover, different methods of manufacturing such as PET for bottles and for PET plates or different types of silicones can be well categorized; see Figure 1 . The various types of silicone dehesives result in well distinguishable, however similar lifetimes $\tau$. The availability of a further criterion for characterization would be of interest. 


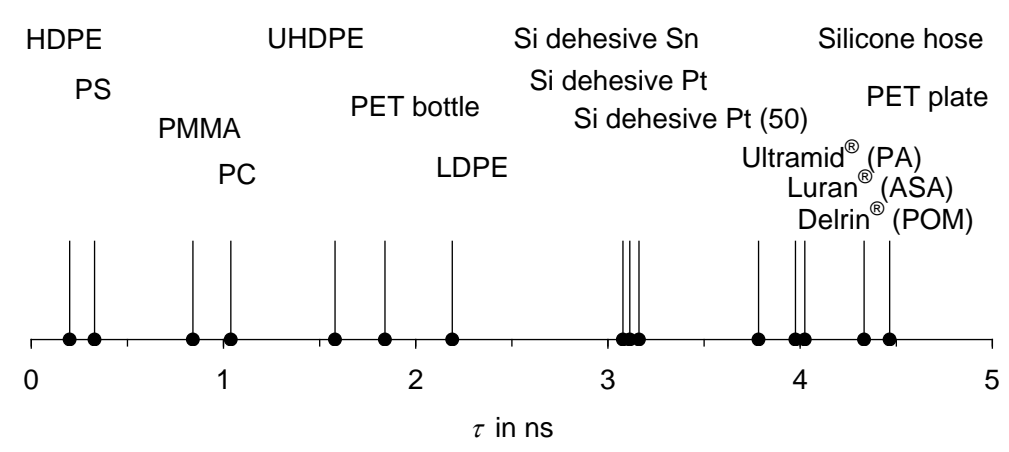

Figure 1. Fluorescence lifetimes $\tau$ in ns of technical polymers. Reproducibility is given within $1 \%$.

\subsection{Bi-Exponential Components of Fluorescence Decay}

We found that the mono-exponential decay dominates resulting in the quite well acceptable first order kinetics. However, there is a bi-exponential component in the decay process. Thus, the lifetimes $\tau$ from Figure 1 mean an averaging of both components depending on their relative contributions. We analyzed the fluorescence decay bi-exponentially and additionally obtained the two time constants $\tau_{1}$ and $\tau_{2}$. All three time constants $\tau$, $\tau_{1}$ and $\tau_{2}$ are reported in Table 1.

The two time constants $\tau_{1}$ and $\tau_{2}$ of the bi-exponential data processing allow the two-dimensional characterization of the polymers as is shown in Figure 2; $\operatorname{Luran}^{\circledR}$ (No. 16) is omitted for clearness because recording the very high value of $\tau_{1}$ would compress the abscissa.

The two-dimensional presentation of the fluorescence lifetimes $\tau_{1}$ and $\tau_{2}$ in Figure 2 allows a clear distinguishing even for similar materials and supplements the $\tau$ values of Figure 1. It becomes simpler to distinguish as well between the widely applied polyethylenes LDPE, HDPE and UHDPE (filled circles) as differently processed materials such as the silicone elastomer Tectosil ${ }^{\circledR}$ (triangles) and PET (squares). The differently processed silicone dehesives (diamonds) become more clearly separated where the catalyst for cross-linking ( $\mathrm{Sn}$ or $\mathrm{Pt}$ ) seems to influence predominantly $\tau_{2}$, whereas the addition of a hardener (Pt (50)) seems to increase $\tau_{1}$. This corresponds to the very low values of $\tau_{1}$ for the elastomer Tectosil ${ }^{\circledR}$.

\subsection{Test for Contaminated PET}

PET is widely applied both for foodstuff such as bottles for water and soft drinks and for technical liquids such as the mineral oils diesel or engine oil. The latter lipophilic liquids can diffuse into the polymeric material such as a plasticizer and would be slowly released. As a consequence, PET for foodstuff must be carefully separated from the latter material. We simulated such contaminations by the contact with the mineral oils diesel and engine oil for one week and purified PET with alkaline detergents according to a technical standard procedure (see materials and methods).

Figure 3 clearly indicates that a pre-treatment of PET results in a lowering of the fluorescence lifetime $\tau$. This is most pronounced for diesel with low molecular weight and low viscosity and slightly less pronounced for engine oil with higher molecular weight and viscosity; this corresponds to observations with silicone dehesives and Tectosil $^{\circledR}$ (see Figure 2). A technical cleaning of the contaminated PET increases the fluorescence lifetime again, however, it does not reach the high value of the neat material by far. Even clean commercial recycling flakes exhibit a slightly diminished fluorescence lifetime.

The detection of contaminations becomes more extended by means of the two-dimensional presentation of the fluorescence lifetimes $\tau_{1}$ and $\tau_{2}$ in Figure 4. The recycling flake with $\tau$ close to the neat material given in Figure 3 becomes much more separated by $\tau_{1}$ and $\tau_{2}$ in Figure 4 . The alterations by means of purifying exhibit different effects for diesel and engine oil. As a consequence, neat material can be well separated.

\subsection{Further Extensions}

Additional optical information about the polymers could be redundantly used for independent characterization of polymers in order to further increase of the reliability or for fine-tuning of the detection concerning the identifi- 


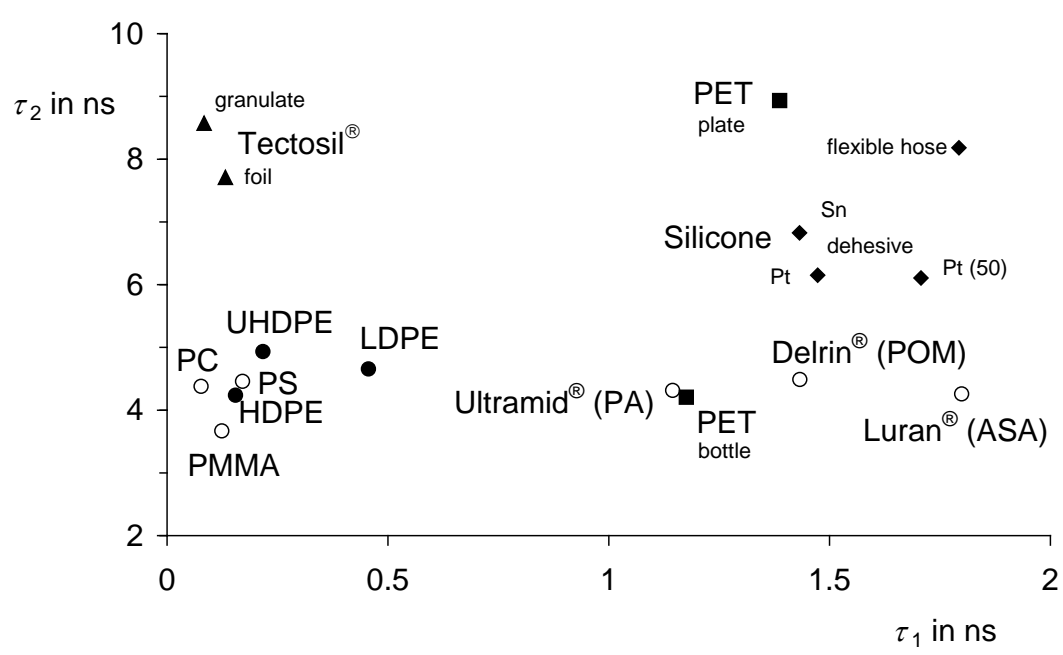

Figure 2. Two-dimensional characterization of polymers by means of their constants $\tau_{1}$ and $\tau_{2}$ of bi-exponential data processing of fluorescence decay. Filled circles: The polyethylenes LDPE, HDPE and UHDPE; squares: PET; diamonds: Silicones; triangles: The silicone elastomer Tectosil ${ }^{\circledR}$.

PET

diesel

recycling flake

diesel, cleaned

engine oil

neat

engine oil, cleaned

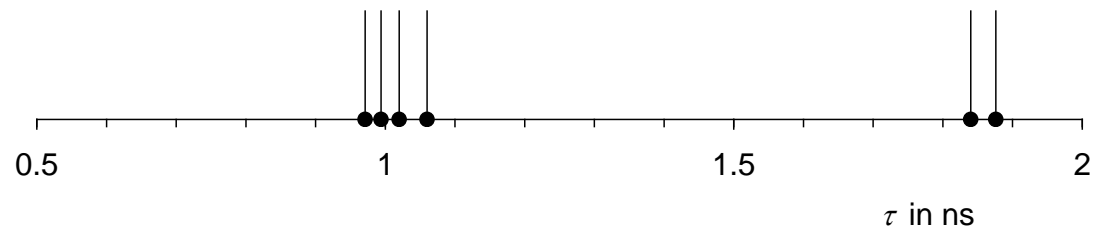

Figure 3. Fluorescence lifetimes $\tau$ of pre-treated PET; expanded range.

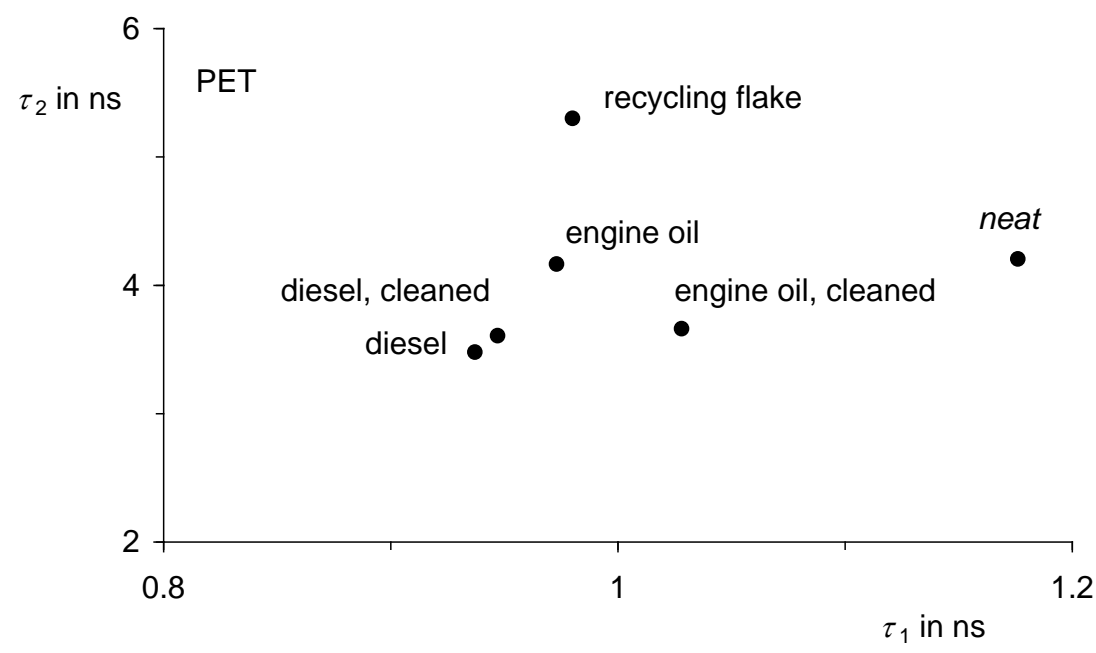

Figure 4. Two-dimensional characterization of PET with various pre-treatment by means of their constants $\tau_{1}$ and $\tau_{2}$ of bi-exponential data processing of fluorescence decay. 
Table 1. Time constants for the autofluorescence decay of technical polymers. For mono-exponential data processing $\tau$ and for bi-exponential $\tau_{1}$ and $\tau_{2}$.

\begin{tabular}{|c|c|c|c|c|}
\hline Polymeric material & $\tau$ & $\tau_{1}$ & $\tau_{2}$ & No. \\
\hline Polymethylmethacrylate (PMMA) & 0.841 & 0.124 & 3.669 & 1 \\
\hline Polystyrene (PS) & 3.290 & 0.171 & 4.457 & 2 \\
\hline Polycarbonate (PC) & 1.038 & 0.077 & 4.379 & 3 \\
\hline Polyethylenterephthalate (PET) bottle ${ }^{\text {a) }}$ & 1.840 & 1.176 & 4.205 & 4 \\
\hline Polyethylenterephthalate (PET) plate & 4.466 & 1.387 & 8.933 & 5 \\
\hline Polyethylene LDPE & 2.19 & 0.456 & 4.655 & 6 \\
\hline Polyethylene HDPE & $<0.2$ & 0.155 & 4.238 & 7 \\
\hline Polyethylene UHDPE & 1.58 & 0.217 & 4.932 & 8 \\
\hline Silicone Tectosil ${ }^{\circledR}$ granulate & & 0.132 & 7.709 & 9 \\
\hline Silicone Tectosil ${ }^{\circledast}$ foil & & 0.084 & 8.572 & 10 \\
\hline Silicone Dehesive Sn & 3.078 & 1.432 & 6.825 & 11 \\
\hline Silicone Dehesive Pt & 3.162 & 1.473 & 6.149 & 12 \\
\hline Silicone Dehesive Pt (50) & 3.114 & 1.707 & 6.106 & 13 \\
\hline Silicone hose & 4.333 & 1.793 & 8.180 & 14 \\
\hline Delrin $^{\circledR}$ (POM) & 4.024 & 1.433 & 4.487 & 15 \\
\hline $\operatorname{Luran}^{\circledR}$ (ASA) & 3.976 & 1.199 & 4.259 & 16 \\
\hline Ultramid $^{\circledR}$ (PA) & 3.784 & 1.145 & 4.313 & 17 \\
\hline
\end{tabular}

a) PET bottle for soft drinks.

cation of certain batches. The fluorescence spectra of polymers are comparably broad and seem to be similar and less characteristic [11] [12]. However, we found that the spectra can be precisely characterized by means of Gaussian functions [14] according to Equation (1) where $I_{(\lambda)}$ is the fluorescence intensity dependent on the wavelength $\lambda$.

$$
I_{(\lambda)}=\sum_{i=0}^{n} I_{i} \cdot e^{-100 \frac{\left(\frac{1}{\lambda}-\frac{1}{\lambda_{i}}\right)^{2}}{2 \sigma_{i}^{2}}}
$$

$I_{i}$ is the intensity of the entire Gaussian band $i, \lambda_{i}$ the position and $\sigma_{i}$ the widths. $\lambda$ is in the denominator because of the reciprocal relation with the energy of the basic Gaussian band. The factor 100 in the exponent simplifies the interconversion between the wavelength in $\mathrm{nm}$ and the energy-linear $\mathrm{kK}\left(10,000 \mathrm{~cm}^{-1}\right)$.

We applied Gaussian analyse to the technical high-performance polymers $\operatorname{Luran}^{\circledR}$ (ASA), Ultramid ${ }^{\circledR}$ (PA) and $\operatorname{Delrin}^{\circledR}$ (POM) as typical examples and found that $n=4$ to 5 Gaussian functions are sufficient for a precise description of the experimental spectra in the visible; see Table 2.

The Fluorescence spectrum of $\operatorname{Luran}^{\circledR}$ in the visible $(>400 \mathrm{~nm})$ is perfectly described by means of four Gaussian bands; see Figure 5. Thus, 12 parameters (see Table 2) are sufficient for the complete description of the broad spectrum and require minimal 12 values [15] of fluorescence intensity at different wavelengths.

Similar results are obtained for Ultramid ${ }^{\circledR}$ where also four Gaussian bands are sufficient for characterization; see Figure 6.

The behaviour of Delrin ${ }^{\circledR}$ is slightly different because of strong fluorescence in the UV close to the visible where five bands concern the visible and one or two bands in the UV should be considered because of tailing into the visible; see Figure 7. 


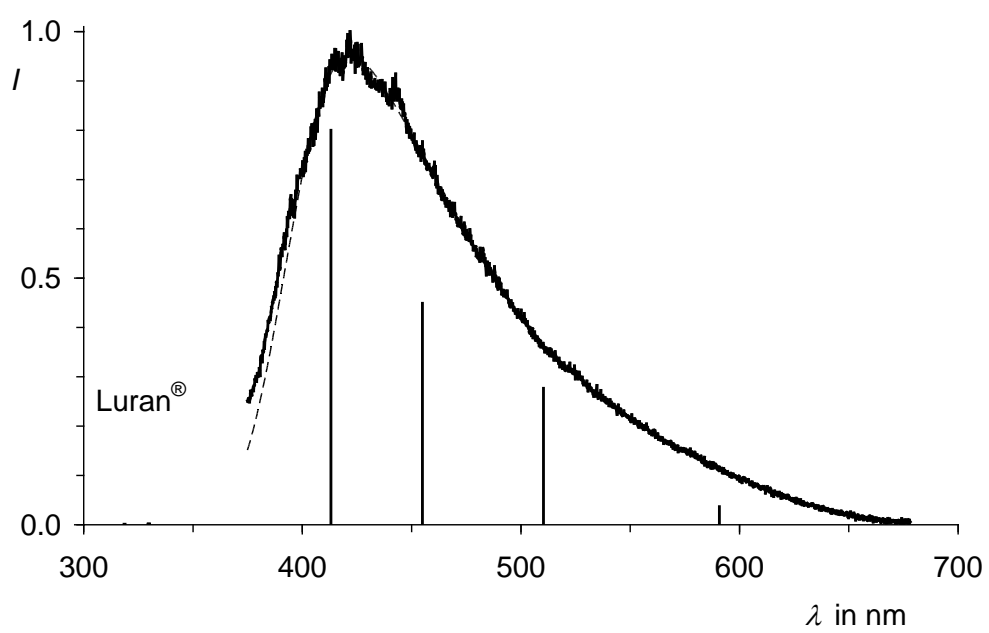

Figure 5. Fluorescence spectrum of Luran ${ }^{\circledR}$ (solid, thick curve) and simulated spectrum (thin, dashed curve, mainly covered by the experimental spectrum) composed of the bands of a Gaussian analysis (>400 $\mathrm{nm})$ on the basis of the parameters of Table 2. Bars: Positions and intensities of the Gaussian bands.

Table 2. Parameter of the sum of Gaussian functions according to Equation (1) for the technical polymers Ultramid ${ }^{\circledR}$ (PA), $\operatorname{Luran}^{\circledR}$ (ASA) und Delrin ${ }^{\circledR}$ (POM). The numbers $i$ of the bands are given in brackets, the wavelengths $\lambda$ in nm and $\sigma$ in kK $\left(10,000 \mathrm{~cm}^{-1}\right)$. The intensities $I$ are derived from the normalized experimental spectrum in the investigated interval.

\begin{tabular}{|c|c|c|c|}
\hline Polymer & Ultramid $^{\circledR}$ & Luran $^{\circledR}$ & Delrin ${ }^{\circledR}$ \\
\hline$\lambda_{\max }(1)$ & 420.93 & 413.08 & 359.96 \\
\hline $2 \sigma^{2}(1)$ & 2.352 & 3.628 & 0.942 \\
\hline$I_{\max }(1)$ & 0.796 & 0.799 & 2.758 \\
\hline$\lambda_{\text {max }}(2)$ & 459.09 & 454.96 & 385.82 \\
\hline $2 \sigma^{2}(2)$ & 2.180 & 3.501 & 1.291 \\
\hline$I_{\max }(2)$ & 0.650 & 0.448 & 0.398 \\
\hline$\lambda_{\text {max }}(3)$ & 499.79 & 510.34 & 418.26 \\
\hline $2 \sigma^{2}(3)$ & 2.369 & 5.630 & 1.115 \\
\hline$I_{\max }(3)$ & 0.161 & 0.276 & 0.242 \\
\hline$\lambda_{\text {max }}(4)$ & 540.03 & 590.78 & 443.58 \\
\hline $2 \sigma^{2}(4)$ & 3.179 & 1.643 & 0.495 \\
\hline$I_{\max }(4)$ & 0.045 & 0.036 & 0.047 \\
\hline$\lambda_{\max }(5)$ & & & 458.34 \\
\hline $2 \sigma^{2}(5)$ & & & 2.591 \\
\hline$I_{\max }(5)$ & & & 0.140 \\
\hline$\lambda_{\max }(6)$ & & & 484.67 \\
\hline $2 \sigma^{2}(6)$ & & & 54.301 \\
\hline$I_{\max }(6)$ & & & 0.013 \\
\hline$\lambda_{\max }(7)$ & & & 504.46 \\
\hline $2 \sigma^{2}(7)$ & & & 1.667 \\
\hline$I_{\max }(7)$ & & & 0.044 \\
\hline
\end{tabular}




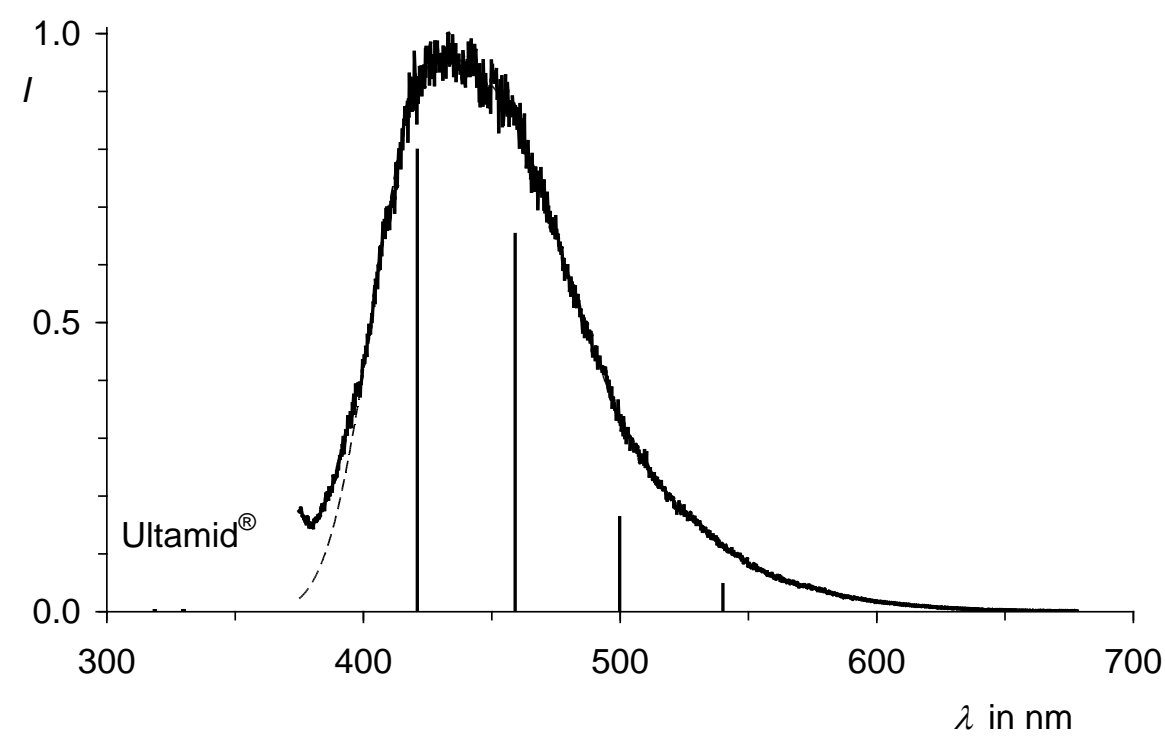

Figure 6. Fluorescence spectrum of Ultramid $^{\circledR}$ (solid, thick curve) and simulated spectrum (thin, dashed curve, mainly covered by the experimental spectrum) composed of the bands of a Gaussian analysis $(>400 \mathrm{~nm})$ on the basis of the parameters of Table 2. Bars: Positions and intensities of the Gaussian bands.

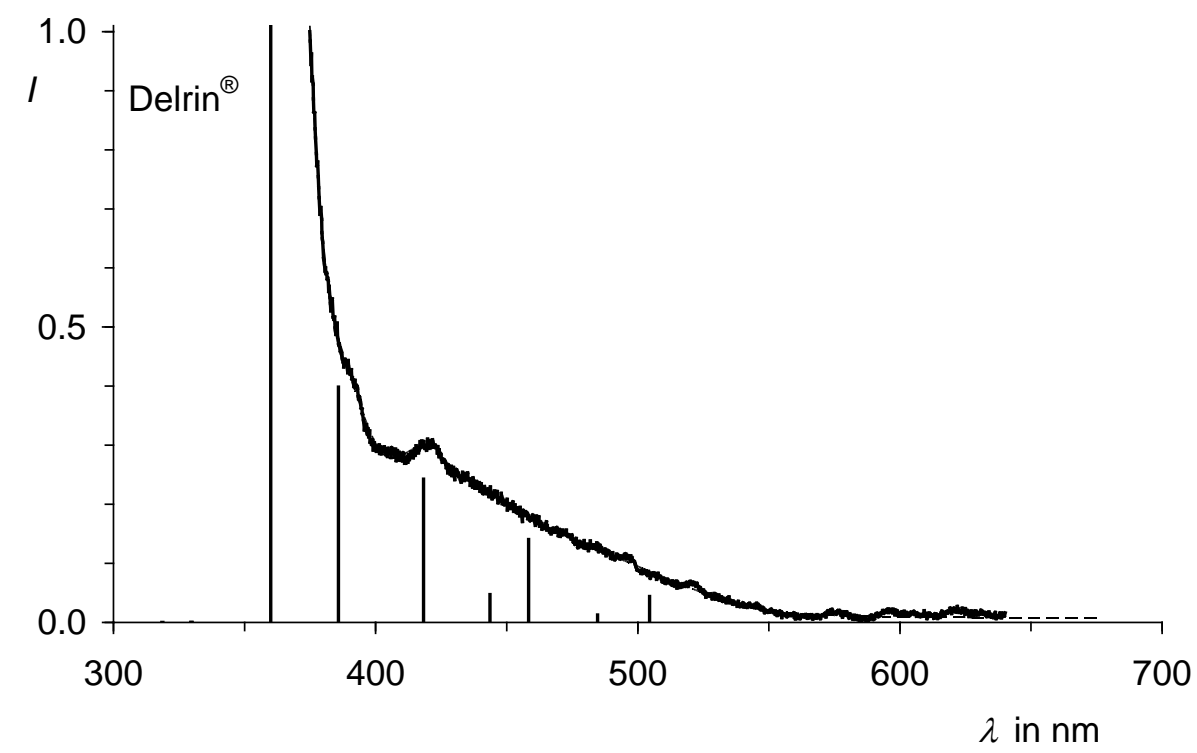

Figure 7. Fluorescence spectrum of Delrin ${ }^{\circledR}$ (solid, thick curve) and simulated spectrum (thin, dashed curve, mainly covered by the experimental spectrum) composed of the bands of a Gaussian analysis $(>375 \mathrm{~nm})$ on the basis of the parameters of Table 2. Bars: Positions and intensities of the Gaussian bands.

\subsection{Practical Setup}

For the determination of the first order time constant it is not necessary to record the whole exponential decay but the sampling of two fluorescence intensities [16]-[18], conveniently before and after the first half life is sufficient; for further details, see ref. [13]. This sampling needs not to be at definite times, but may be obtained by integration over a defined interval and thus, increases the signal to noise ratio. Moreover, an absolute determination of intensities is not necessary because of unproblematic calibration. Four points are necessary for bi-exponential decays and for some cases three would be enough. 
As a consequence, a convenient setup is the application of a laser with ns or sub ns light pulses for optical excitation and phase shifted detections of the fluorescence intensities; two detections are necessary for mono-exponential decay and four for bi-exponential. A spectral resolution to 12 until 15 wavelengths allows the further identification by means of the data of the Gaussian analyses. The optical detection can be accumulated for the improvement of the signal to noise ratio where a frequency of repetition of $15 \mathrm{MHz}$ may be conveniently reached. Thus, half a ton of material can be separated per hour by the application of convenient techniques.

\section{Conclusion}

Technical polymers can be identified by means of the mono-exponential lifetime of their autofluorescence. A biexponential recording allows an advanced classification useful for the discrimination of chemical similar materials such as LDPE, HDPE and UHDPE, different processing or the recognition of contaminates. The fluorescence spectra of polymers can be simplified by means of Gaussian analyses and applied as a source for redundant information of fine tuning for the recognition of certain batches. The optical method allows automation for sorting plastics in the dimensions of half a ton per hour.

\section{Acknowledgements}

This work was supported by The BMBF, CIPSM cluster in Munich, and the Fonds der Chemischen Industrie. We thank Dr. Moritz Ehrl for technical assistance. We thank Karin Haslböck and Dipl.-Ing. Dipl.-Wl-Ing. Thomas Böhme for material support.

\section{References}

[1] Nemeth, E., Schubert, G., Albrecht, V. and Simon, F. (2005) The Triboelectric Charging of Mixtures of Plastics in the Technology of Recycling. Aufbereitungs-Technik, 46, 35-46.

[2] Nemeth, E., Simon, F., Albrecht, V. and Schubert, G. (2006) Separating a Mixture of Plastics Comprises Electrostatic Sorting after Plasma Treatment and Triboelectric Charging. Ger. Patent, DE 102004024754 B3 (May 12. 2004); Chemical Abstracts, 144, Article ID: 392348.

[3] Gohs, U., Albrecht, V., Husemann, K., Reinsch, E., Schuenemann, R. and Simon, F. (2009) Waste Plastic Mixture i.e. Completely Crushed Polyethylene- and Polypropylene-particles, Separation Method for e.g. Electrical Industry, Involves Subjecting Plastic Mixture to Ttriboelectric Charging, and Separating Pure Polyolefin Particles. German Patent Application, DE 102007055765 A1 (Dec. 11, 2007); Chemical Abstracts, 151, Article ID: 57663.

[4] Huth-Fehre, Th., Feldhoff, R., Kantimm, Th., Quick, L., Winter, F., Cammann, K., van den Broek, W., Wienke, D., Melssen, W. and Buydens L. (1995) NIR-Remote Sensing and Artificial Neural Networks for Rapid Identification of Post Consumer Plastics. Journal of Molecular Structure, 348, 143-146. http://dx.doi.org/10.1016/0022-2860(95)08609-Y

[5] Michaeli, W., Plessmann, K.W., Andrassy, B., Breyer, K. and Laufens, P. (1998) Qualitative and Quantitative Characterisation of Mixed Polymers Using Near-Infrared-Spectroscopy (NIR). Polymer Recycling, 3, 287-293; Chemical Abstracts, 132, Article ID: 181307.

[6] Corbet, E.C., Frey, J.G., Groce, R.I. and Hendra, P.J. (1994) An Investigation into the Applicability of Luminescent Tagging to Polymer Recovery. Plastics, Rubber and Composite Processing and Application, 21, 5-11.

[7] Rafi Ahmad, S. (2000) Marking of Products with Fluorescence Tracers in Binary Combinations for Automatic Identification and Sorting. Assembly Automation, 20, 58-65. http://dx.doi.org/10.1108/01445150010311617

[8] Alam, M.K., Stanton, S.L. and Hebner, G.A. (1994) Near Infrared Spectroscopy and Neural Network for Resin Identification. Spectroscopy, 9, 31-39.

[9] Scott, D.M. (1995) A Two-Colour Near-Infrared Sensor for Sorting Recycled Plastic Waste. Measurement Science and Technology, 6, 156-159. http://dx.doi.org/10.1088/0957-0233/6/2/004

[10] General Electric Company (inv. Hubbard, S., Potyrailo, R., Schottland, P. and Thomas, V.) (2005) Tagging Materials for Polymers, Methods, and Articles Made Thereby. US-Patent, 2005/0095715.

[11] Langhals, H., Schmid, T., Herman, M., Zwiener, M. and Hofer, A. (2013) Binary Fluorescence Labeling for the Recovery of Polymeric Materials for Recycling. International Journal of Science, Environment and Technology, 7, 124132.

[12] Langhals, H., Schmid, T., Herman, M., Zwiener, M. and Hofer, A. (2012) Marking of Polymer Materials with Fluorescence Dyes for Their Clear Automatic Sorting. German Patent Application, DE 102012012772.3 (June 22, 2012); 
Chemical Abstracts, 160, Article ID: 63983.

[13] Langhals, H., Zgela, D. and Schlücker, T. (2014) High Performance Recycling of Polymers by Means of Their Fluorescence Lifetimes. Green and Sustainable Chemistry, 4, 144-150. http://dx.doi.org/10.4236/gsc.2014.43019

[14] Langhals, H. (2002) The Rapid Identification of Organic Colorants by UV/Vis-Spectroscopy. Analytical and Bioanalytical Chemistry, 374, 573-578. http://dx.doi.org/10.1007/s00216-002-1473-x

[15] Langhals, H. (1982) The Determination of the Composition of Binary Liquid Mixtures by Means of Fluorescence Measurements. Fresenius' Zeitschrift für Analytische Chemie, 310, 427-428. http://dx.doi.org/10.1007/BF00483019

[16] Ballew, R.M. and Demas, J.N. (1989) An Error Analysis of the Rapid Lifetime Determination Method for the Evaluation of Single Exponential Decays. Analytical Chemistry, 61, 30-33. http://dx.doi.org/10.1021/ac00176a007

[17] Woods, R.J., Scypinski, S., Cline Love, L.J. and Ashworth, H.A. (1984) Transient Digitizer for the Determination of Microsecond Luminescence Lifetimes. Analytical Chemistry, 56, 1395-1400. http://dx.doi.org/10.1021/ac00272a043

[18] Meier, R.J., Fischer, L.H., Wolfbeis, O.S. and Schäferling, M. (2013) Referenced Luminescent Sensing and Imaging with Digital Color Cameras: A Comparative Study. Sensors and Actuators, B177, 500-506. http://dx.doi.org/10.1016/j.snb.2012.11.041 